\title{
Determinantes sociales, económicos y ambientales de envejecimiento activo en ancianos atendidos en un centro de atención integral a la salud
} Social, economic and environmental determinants of active aging in the elderly attended in a center of integral health care

\section{Determinantes sociais, econômicos e ambientais de envelhecimento ativo em idosos atendidos em um centro de atenção integral à saúde}

\begin{abstract}
Sanni Moraes de Oliveira ${ }^{1}$, KeyllaTalitha Fernandes Barbosa ${ }^{2}$, Fabiana Maria Rodrigues Lopes de Oliveira ${ }^{3}$,
Wiliana Aparecida Alves de Brito Fernandes ${ }^{4}$, Maria das Graças Melo Fernandes ${ }^{5}$

1,2,3,4,5 Enfermeira, Universidade Federal da Paraíba, João Pessoa, Paraíba, Brasil.

Cómo citar este artículo en edición digital: Oliveira, S.M., Barbosa, K.T.F., Oliveira, M.R.L., Fernandes, W.A.A.B., \& Fernandes, M.G.M. (2017). Determinantes sociales, económicos y ambientales de envejecimiento activo en ancianos atendidos en un centro de atención integral a la salud. Cultura de los Cuidados (Edición digital), 21(49).

Recuperado de http://dx.doi.org/10.14198/cuid.2017.49.17
\end{abstract}

Correspondencia: Remitirse al correo electrónico. Correo electrónico: sannidsm@gmail.com

Recibido: 12/02/2017; Aceptado: 20/05/2017

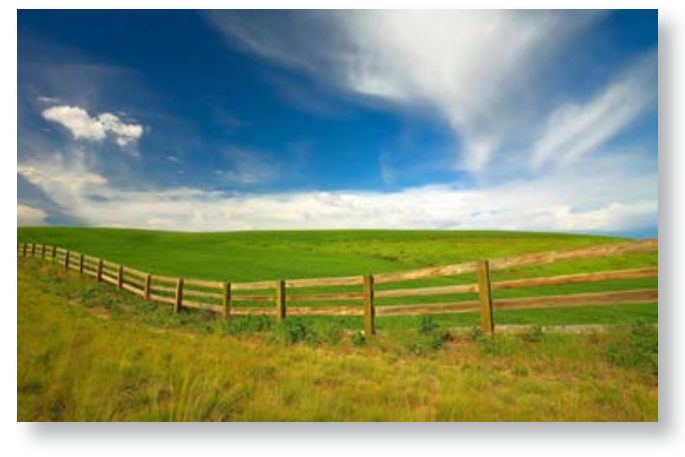

ABSTRACT

The aim of this study is to evaluate social, economic, and environmental determinants of active aging in older adults assisted at the Center for Integrated Health Care to elderly in João Pessoa, Paraiba. It is a descriptive, transversal study, with a qualitative approach. The target population consisted all older adults $(n=224)$ assisted at the Center for Integrated Health Care. Data collection was conducted through semi-structured interview guide in the period from October to December of 2014. The majority of older adults were among the age group of 60 to 69 years old, and the most of them were female. A number of years of study were between 4 to 8 . The vast majority of this population does not have a paid work, and they receive a monthly income between 1 to 3 minimum wage. The most predominant source of social benefit was retiring. The theft was the type of violence the most experienced. The most of them live in their own house with a good physical structure and basic sanitation, but these homes have risks to cause accidental fall. This study is important for reflection and attitude towards of health professionals to improve care for the elder population. Therefore, the care for elderly can understand their necessities and promote autonomy emphasizing social, economic, and environmental determinants of active aging.

Keywords: Nursing, aging, health of the elderly, social determinants of health.

\section{RESUMEN}

Se ha partido del objetivo de evaluar los determinantes sociales, económicos y ambien- 
tales de envejecimiento activo entre ancianos asistidos en un Centro de Atención Integral a la Salud del Anciano en João Pessoa - Paraíba. Estudio descriptivo, transversal, con planteamiento cuantitativo. La población incluyó todos los ancianos atendidos en el mencionado servicio. La muestra estaba compuesta por 224 ancianos. Los datos fueron recolectados mediante entrevistas estructuradas, efectuadas entre octubre y diciembre 2014. Se encontró un predominio del grupo de edad de 60 a 69 años, en su mayoría mujeres. El promedio de años de escolaridad fue de 4 a 8, la mayoría no tiene trabajo remunerado, posee un ingreso mensual de 1 a 3 salarios mínimos, $y$ la jubilación fue la fuente de beneficio social más prevalente. El robo fue la violencia más experimentada. La mayoría habitaba en sus propias viviendas con buena cobertura de saneamiento básico, pero con condiciones favorables para la ocurrencia de caídas. Así, los resultados encontrados constituyen un producto valioso para la reflexión y la toma de actitud de profesionales volcadas hacia la atención con el fin de promover la autonomía del anciano y una mejor comprensión de las necesidades individuales, señalando los factores sociales, económicos y ambientales del envejecimiento activo.

Palabras clave: Enfermería, envejecimiento, salud del anciano, determinantes sociales de la salud.

\section{RESUMO}

Objetivou-se avaliar os determinantes sociais, econômicos e ambientais de envelhecimento ativo entre idosos assistidos em um Centro de Atenção Integral à Saúde do Idoso no município de João Pessoa - Paraíba. Estudo descritivo, transversal, de abordagem quantitativa. A população compreendeu todos os idosos atendidos no referido serviço. A amostra foi composta por 224 idosos. Os dados foram coletados mediante entrevista estruturada, realizado no período de outubro a dezembro de 2014. Verificou-se predominância da faixa etária de 60 a 69 anos, sendo a maioria do sexo feminino. A média de anos de estudo foi 4 a 8 , a maioria não exerce trabalho remunerado, possui renda mensal de 1 a 3 salários mínimos, sendo a aposentadoria a fonte de benefício social mais prevalente. $\mathrm{O}$ roubo foi o tipo de violência mais vivenciado. A maioria residia em casas próprias com boa cobertura de saneamento básico, e condições favoráveis para ocorrência de quedas. Deste modo, os resultados deste estudo, constituem valioso produto para reflexão e tomada de atitude de profissionais voltadas para o cuidado que vise a promoção da autonomia do idoso e melhor compreensão das necessidades individuais enfatizando os determinantes sociais, econômicos e ambientais do envelhecimento ativo.

Palavras-chave: Enfermagem, envelhecimento, saúde do idoso, determinantes sociais da saúde.

\section{INTRODUÇÃO}

O envelhecimento da população é um fenômeno mundial, apontado como um dos fatos demográficos mais relevantes nas últimas décadas. É possível inferir que o ritmo do envelhecimento tem sido sistemático e consistente, e a perspectiva é que em 2025, o Brasil deverá ter $15 \%$ do seu contingente populacional constituído por idosos, ocupando o sexto lugar dentre os países mais envelhecidos no mundo (IBGE, 2013; SDH, 2012; IBGE, 2012).

$\mathrm{O}$ processo do envelhecer, traz consigo a diminuição gradual da capacidade funcional, a qual é progressiva e aumenta com a idade. 
Por diversas vezes, esta diminuição resulta em grandes prejuízos à saúde do idoso, como a incapacidade funcional e dependência, que ocasionam restrição e perda de habilidades, além de dificuldade ou incapacidade de executar atividades relacionadas à vida diária (Ferreira et al., 2012).

Esta tendência implica em novas demandas para as políticas públicas, tal qual, seguridade social, e assistência integralizada à pessoa idosa. Quanto as novas reflexões acerca do envelhecimento, no que concerne aos projetos político-sociais, econômicos, culturais e educacionais desenvolvidos pela sociedade em geral, existe um direcionamento para mudança de paradigmas com vistas a estimular o envelhecimento saudável e ativo (Santos; Paes, 2014; Ribeiro; Paúl, 2011). Nessa perspectiva, o envelhecimento ativo é definido como o processo de otimização das oportunidades de saúde, participação e segurança, com o objetivo de melhorar a qualidade de vida à medida que as pessoas ficam mais velhas. Configurando-se como o equilíbrio biopsicossocial e integral de um ser humano que está arraigado a um contexto social e que tem a possibilidade de desenvolver suas inúmeras potencialidades (Ferreira et al., 2010). A Organização Mundial da Saúde (2005) define como principais determinantes para o envelhecer ativo os seguintes fatores: serviços sociais e de saúde; determinantes comportamentais; determinantes pessoais; ambiente físico; determinantes sociais e; determinantes econômicos. As evidências sugerem que todos os determinantes, bem como a interação entre eles refletem no envelhecimento dos indivíduos e populações (Vicente \& Santos, 2013).Destaca-se, no âmbito desse estudo, a relevância dos determinantes sociais, econômicos e ambientais para que a pessoa idosa tenha um processo de envelhecimento saudável. Neste contexto, os determinantes sociais incluem o apoio social, as oportunidades de educação, a paz e a proteção contra a violência e os maus tratos. Os determinantes econômicos englobam três aspetos relevantes: o rendimento, a proteção social e o trabalho remunerado ou não. Já os fatores relacionados com o ambiente físico, estes podem marcar a diferença entre a independência e a dependência, pois são importantes para determinar o bem-estar e qualidade de vida da pessoa idosa (Azevedo, 2015).

Diante do exposto, tornou-se necessário investigar a expressão dos determinantes do envelhecimento ativo, tendo em vista que, ainda são incipientes os estudos, especialmente no cenário local. Pretende-se que, os dados empíricos apreendidos neste estudo possam contribuir para uma assistência social, de saúde efetiva e holística a pessoa idosa, atentando para as suas necessidades específicas, pautada na valorização do envelhecimento da população de forma ativa e saudável.

Desse modo, foi formulada a seguinte questão norteadora para guiar o presente estudo: quais os determinantes sociais, econômicos e ambientais do envelhecimento ativo são evidenciados por idosos assistidos em um Centro de Atenção Integral à Saúde do Idoso? Para responder tal questionamento, foi delimitado o seguinte objetivo: avaliar os determinantes sociais, econômicos e ambientais de envelhecimento ativo entre idosos assistidos em um Centro de Atenção Integral à Saúde do Idoso no município de João Pessoa - Paraíba.

\section{METODOLOGIA}

Trata-se de um estudo descritivo, do tipo transversal, desenvolvido com pessoas idosas assistidas no Centro de Atenção Integral à Saúde do Idoso (CAISI) do município de João 
Pessoa, Paraíba. A determinação da amostra foi do tipo probabilística, por meio da técnica de amostragem simples. Para a seleção dos idosos investigados, foi levantado junto ao serviço a quantidade de idosos assistidos. Posteriormente, delimitou-se a amostra considerando a seguinte fórmula: $\mathrm{n}=\mathrm{Z} 2 \mathrm{PQ} / \mathrm{d} 2$, sendo $\mathrm{n}$ = tamanho amostral mínimo; $\mathrm{Z}=$ variável reduzida; $\mathrm{P}=$ probabilidade de encontrar o fenômeno estudado; $\mathrm{Q}=1-\mathrm{P} ; \mathrm{d}=$ precisão desejada. Adotou-se $\mathrm{p}=50 \%$, por se tratar de uma avaliação multidimensional, e parâmetro de erro amostral de $5 \%$.

Participaram do estudo indivíduos com sessenta anos ou mais, de ambos os sexos, que após devidamente esclarecidos dos propósitos e passos operacionais, aceitaram participar da pesquisa, totalizando a amostra de 224 idosos. A coleta de dados foi realizada no período de outubro a dezembro de 2014, mediante entrevista subsidiada por um instrumento estruturado, contemplando questões pertinentes ao objetivo proposto para o estudo. Este instrumento compreendeu a avaliação multidimensional do envelhecimento ativo, conforme proposto por Vicente (2012), enfatizando os determinantes sociais, econômicos e ambientais.

A análise dos dados foi efetivada por meio de abordagem quantitativa através de estatística descritiva de natureza univariada para todas as variáveis, incluindo medidas de frequência, de posição e dispersão. Para comparação das principais variáveis categóricas, foi utilizado o Teste do Qui-quadrado. Para tanto, utilizou-se o sistema computacional Statistical Package for the Social Sciences - SPSS versão 20.0, por ser adequada ao alcance dos objetivos do estudo e por possibilitar a precisão e generalização dos seus resultados.

Cabe destacar que, durante todo o processo da pesquisa, especialmente na fase da coleta de informações empíricas, foram observados os aspectos éticos que normatizam a pesquisa envolvendo seres humanos dispostos na Resolução 466/12 do CNS/MS/BRASIL, após a aprovação de projeto pelo Comitê de Ética em Pesquisa da Universidade Federal da Paraíba, sob protocolo 0384/14 e CAAE $n^{\circ}$. 33089014.5.0000.5188 de 21 de agosto de 2014 .

\section{RESULTADOS}

Evidenciou-se que a faixa etária predominante entre a população estudada foi de 60 a 69 anos $(60,3 \%)$, sendo a maioria pessoas do sexo feminino $(68,3 \%)$, que se autodeclaram de cor parda $(41,1 \%)$, que eram casados $(39,7 \%)$ e católicos $(66,1 \%)$ (Tabela 1$)$.

Em relação aos determinantes econômicos, $192(85,7 \%)$ possuíam renda mensal entre 1 a 3 salários mínimos, 145 (64,7\%) informaram que recebiam algum provento da previdência social, 113 (50,4\%) exerciam algum trabalho não remunerado em sua residência, tais como: jardim e/ou horta 78 (34,8\%); cuidador de netos e/ou outros familiares $54(24,1 \%)$ e; também trabalho voluntário em igreja e/ou escolas 39 (17,4\%), conforme Tabela 2.

No contexto dos determinantes sociais, a maioria dos investigados relatou ter frequentado escola de quatro a oito anos, totalizando $77(34,4 \%)$ idosos, $83(37,1 \%)$ relataram ter sofrido violência do tipo roubo. Em relação ao convívio familiar, 179 (79,9\%) residiam com familiares. Quanto às redes de apoio sociais, 149 (66,5\%) frequentavam igreja e/ou grupo religiosos (Tabela 3).

No que se refere aos determinantes do envelhecimento ativo relacionados ao ambiente físico, 220 idosos $(98,2 \%)$ tinham suas residências abastecidas com água da Companhia de Água e Esgotos da Paraíba (CAGEPA), dos quais $123(54,9 \%)$ possuía rede de esgoto. O 
lixo era coletado pela Empresa Municipal de Limpeza Urbana (EMLUR) em 207 (92,4\%) domicílios. Além disso, 181 (80,8\%) eram proprietárias de suas residências e possuíam quarto privativo para o idoso. Quanto à avaliação de riscos de quedas para os idosos em suas moradias, $63(28,1 \%)$ relataram presença de degraus ou escadas e $53(23,7 \%)$ usam tapetes (Tabela 4).

\section{DISCUSSÃO}

Ao analisar os dados do estudo segundo faixa etária, observa-se que a maior parte dos entrevistados, são considerados idosos jovens, com idade entre 60 a 69 anos, havendo uma diminuição gradativa de indivíduos nas faixas subsequentes, especialmente a partir dos 80 anos. Esses achados podem estar atrelados ao fato do estudo ter sido desenvolvido a nível ambulatorial, assim quanto mais jovem maior a possibilidade do idoso sair de seus domicílios para procurar assistência à saúde.

Com relação ao sexo, evidenciou-se a maioria sendo do sexo feminino, o que demonstra um fenômeno já bem discutido na literatura gerontológica, denominado feminização do envelhecimento. O sexo feminino está mais atrelado ao cuidado à saúde ao longo do ciclo vital, assim como, a uma maior prática preventiva e uma menor exposição a situações de risco, ocupacionais ou de estilo de vida. Isto lhes confere um envelhecer mais ativo e saudável, quando comparado ao sexo oposto (Oliveira et al., 2013).

Quanto à religiosidade, identificou-se predominância do catolicismo, tal fato traz reflexões que perpassam a cultura e história brasileira (Baricelli et al., 2012). Farias e Santos (2012), afirmam que o constructo da religiosidade é mais comum entre idosos do que em qualquer outra idade e mesmo que apresentem limitações físicas para continuarem frequentando espaços religiosos, estes costumam permanecer firmes em suas crenças, constituindo importante rede de apoio, e consequências positivas para o envelhecimento saudável. Tal aspecto é comprovado quando a maioria dos idosos entrevistados destacam a igreja como principal fonte de apoio.

Assim, a fé representa um papel significativo frente aos desafios cotidianos geradores de estresse, sendo considerada como um recurso cognitivo, emocional e comportamental, propiciando maiores condições ao indivíduo idoso de lidar com as perdas, a dependência e a tendência ao isolamento. Ademais, pode ser entendida também como uma motivação para a busca de um sentido para a vida. Neste contexto, ressalta-se que tais aspectos contribuem para o alcance de um envelhecimento bem-sucedido (Baricelli et al., 2012; Farias; Santos, 2012; Galicioli; Lopes; Rabelo, 2012).

No tocante ao estado civil, este estudo aponta uma prevalência de idosos casados e viúvos. Isto desvela uma questão de gênero, uma vez que, culturalmente, as mulheres têm uma maior tendência de ficarem viúvas enquanto que os homens têm uma maior taxa de recasamento, aumentando a proporção de casados e viúvos nessa faixa etária (Barbosa, 2015; Silva; Menandro, 2014; IBGE, 2011). Verificou-se que a grande maioria dos investigados coabitam com familiares. A família é vista como o melhor local para o envelhecimento saudável, pois constitui uma importante rede de apoios as pessoas idosas, garantir-lhes autonomia, identidade, dignidade e interação social. Entretanto, é oportuno salientar que esse contexto merece atenção para que a coabitação com o idoso e o consequente aumento de responsabilidades e tarefas dos membros familiares, não seja atribuído a um efeito nefasto sobre o 




relacionamento familiar, e resulte na desvalorização do papel da pessoa idosa na família (Gonçalves et al., 2011; Farias; Santos, 2012; Sebastião; Albuquerque, 2011).

Corroborando com isso, o fato de muitos idosos residirem com familiares pode, também, ser alternativa à falta de recursos financeiros dos filhos para a manutenção de moradias próprias. $\mathrm{O}$ rendimento associado ao fato de o idoso possuir casa própria, tem proporcionado a ele maior capacidade de suporte familiar, fazendo com que, muitos filhos e netos tornem-se dependentes financeiramente, culminando em abdicação do seu salário em prol da garantia do sustento de seus familiares, sendo estes responsáveis por mais de $90 \%$ do total do rendimento mensal do domicílio, em consequência, principalmente, de suas aposentadorias (Araújo et al., 2012; Geib, 2012; IBGE, 2011).

Neste contexto, no que se refere à renda mensal, verificou-se que a maioria dos indivíduos deste estudo possuem rendimento de um a três salários mínimos, provenientes principalmente da aposentadoria. As alterações fisiológicas inerentes ao envelhecimento, culminam em grandes dificuldades de permanecer no mercado de trabalho, assim, os idosos passam a depender de fontes de renda com remunerações baixas, a exemplo de aposentadorias e pensões. Esses mesmos fatores físicos e funcionais, impulsionam os idosos a não realizarem trabalhos remunerados. Pesquisas demonstram que essas dificuldades podem ocasionar condições de dependência financeira e social revelando um prejuízo no poder aquisitivo, que aliado às necessidades especiais e os altos gastos com a saúde e sobrevida, influenciam na possibilidade de consumo e consequentemente na qualidade de vida da pessoa idosa (Farias; Santos, 2012; Deponti; Acosta, 2010; Sthal; Berti; Palhares, 2010).

Em contrapartida, o presente estudo demonstrou que muitos idosos entrevistados exercem alguma atividade não remunerada, sendo em sua própria residência, ou mesmo fora dela, como cuidadores ou exercendo alguma atividade voluntária na sociedade. Sabe-se que a atuação em trabalhos voluntários, principalmente para nesta população, serve como alternativa para promoção da saúde, devido ao prestígio social e sustentação da autonomia, sendo considerado uma ferramenta eficaz para $o$ alcance de um envelhecimento ativo e saudável. Destarte, ao se manterem socialmente ativos, os idosos afastam-se do preconceito, socialmente imposto, de que o envelhecimento está atrelado à inutilidade do indivíduo (Reis et al., 2014).

Quanto ao grau de instrução, prevaleceram os idosos com baixa escolaridade, os quais declararam ter apenas o primário. Tal fato reflete nas políticas de educação e desigualdades sociais que imperavam em décadas pregressas, onde os obstáculos enfrentados para ter acesso à educação eram maiores. Logo, como a educação e a alfabetização são essenciais ao longo de todo o ciclo de vida, a sua ausência vai ter repercussões no processo do envelhecimento, na qualidade de vida, na adesão de práticas saudáveis, na adaptação com o mundo e no empoderamento da pessoa idosa diante seus 
direitos (Azevedo, 2015; Fernandes; Garcia, 2010; Braga et al., 2010).

Outro determinante social inerente ao envelhecimento ativo está relacionado a violência e maus tratos exercidos contra os idosos. Os achados desta pesquisa trazem o roubo e o abandono por parte da família. O idoso é visto como um indivíduo fragilizado e impotente diante da sociedade, e por vezes, dos familiares, sendo, portanto, alvo de um elevado número de maus tratos. No Brasil, ainda não se tem ideia da prevalência e dimensão real destes problemas, no entanto, as características da sociedade brasileira atual, que desvela um forte preconceito contra o envelhecimento associado ao culto à juventude, favorecem consideravelmente para disseminação da violência contra a pessoa idosa. (Duque et al., 2012).

No tocante aos determinantes de ambiente físico, o estudo encontrou que muitos dos entrevistados residiam em casas próprias, abastecidas de água tratada, saneamento básico e com boa cobertura de coleta de resíduos, no entanto apresentavam condições domiciliares favoráveis a ocorrência de quedas. Sabe-se que as características da comunidade, correspondem às condições de vida, sendo determinantes de saúde e deste modo, deve ser compreendido como um direito dos cidadãos e um item imprescindível para a qualidade de vida e processo de envelhecer saudável (Barbosa, 2015, Sant'anna, 2010).

As condições de moradia estão fortemente relacionadas com a ocorrência de queda em idosos, por se constituírem como riscos ambientais que interferem na realização das atividades de vida diária. É comum que, o domicílio do idoso, por ser um ambiente familiar, se torne muitas vezes um lugar de risco, pois a autoconfiança causada pelo conhecimento do local diminui a atenção (Nogueira et al.,
2012). Os fatores ambientais mais associados à ocorrência de quedas são: iluminação inadequada, superfícies escorregadias, tapetes altos ou estreitos, obstáculos físicos, calçados e roupas inadequadas, e irregularidades no solo. A elevada incidência das quedas na população idosa pode gerar prejuízos físicos, declínio ou perda da capacidade funcional, insegurança, isolamento social e até mesmo a morte, além dos elevados custos para o sistema de saúde. (Fernandes et al., 2014, Traldi, 2013).

Os achados da presente pesquisa confirmam que, para ser bem-sucedido, o envelhecimento deve envolver diversos fatores que se interelacionam, seja na manutenção da capacidade funcional, autonomia financeira, afeição familiar, convívio social, condições ambientais sendo fundamental a sua combinação com o engajamento ativo com a vida, o que representa o conceito mais amplo do envelhecer bem. Neste contexto, o envelhecimento ativo deve ser considerado como uma condição a ser atingida por quem lida com as mudanças inerentes ao envelhecer. (Oliveira; Menezes, 2011; Ferreira et al., 2012).

\section{CONCLUSÃO}

Os resultados deste estudo, constituem valioso produto para reflexão e tomada de atitude de profissionais voltadas para o cuidado que vise a promoção da autonomia da pessoa idosa e melhor compreensão das necessidades individuais dos mesmos, assim como a promoção de novos diálogos sobre envelhecimento e estímulo a medidas que façam dos indivíduos atores no processo de envelhecimento saudável e ativo.

O objetivo do estudo em identificar os fatores determinantes de envelhecimento relacionados aos fatores sociais, econômicos e ambientais, foi alcançado, no entanto, destaca-se 


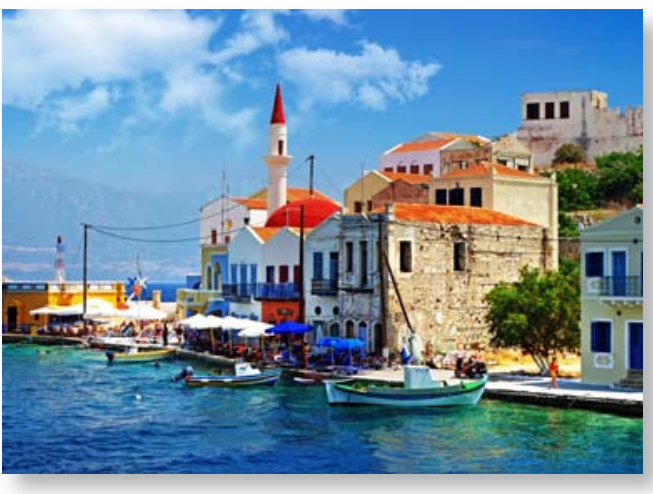

como limitações do estudo: não verificação se os idosos investigados são ativos ou passivos neste processo de envelhecimento humano, como também, a não avaliação do grau de dependência ou autonomia desses idosos, visto que o instrumento utilizado é insuficiente para essa determinação. Assim, sugere-se a realização de novos estudos que utilizem uma abordagem multidimensional do envelhecimento ativo. Espera-se que esta pesquisa sirva como embasamento para futuros estudos envolvendo os determinantes sociais, econômicos e ambientais com vistas a aumentar o conhecimento na temática abordada.

\section{REFERÊNCIAS:}

- Araújo, C. K., Cardoso, C. M. C., Moreira, E. P., Wegner, E. \& Areosa, S.V.C. (2012) Vínculos familiares e sociais nas relações dos idosos. Revista Jovens Pesquisadores, 1, 97-107.

- Azevedo, M. S. A. (2015). O envelhecimento ativo e a qualidade de vida: uma revisão integrativa. (Dissertação não publicada). Escola Superior de Enfermagem do Porto, Porto.

- Barbosa, K. T. F. (2015). Vulnerabilidade física, social e programática de idosos atendidos na atenção primária de saúde do Município de João Pessoa, Paraíba. (Dissertação não publicada). Universidade Federal da Paraíba, Paraíba.

- Barricelli, I. L. F. O. B. L., Sakumoto, I. K. Y., Silva, L. H. M. \& Araújo, C. V. (2012). Influência da orientação religiosa na qualidade de vida de idosos ativos. Revista Brasileira de Geriatria e Gerontologia, 15(3), 505-515.

- Braga, L. S., Macinko, J., Proietti, F. A., César, C. C. \& Lima-Costa, M. F. (2010). Diferenciais interurbanos de vulnerabilidade da população idosa. Caderno de Saúde Pública, 26(12), 2307-2315.

- Cruz, R. C. \& Ferreira, M. A. (2011). Um certo jeito de ser velho: representações sociais da velhice por familiares de idosos. Texto Contexto Enfermagem, 20(1), 144151.

- Deponti, R. N. \& Acosta, M. A. F. (2010). Compreensão dos idosos sobre os fatores que influenciam no envelhecimento saudável. Estudos Interdisciplinares sobre o Envelhecimento, 15(1), 33-52.

- Duque, A. M., Leal, M. C. C., Marques A. P. O. \& Eskinazi, F. M. V. (2012). Violência contra idosos no ambiente doméstico: prevalência e fatores associados (Recife/PE). Ciência e Saúde Coletiva, 17(8), 2199-2208.

- Farias, R. G. \& Santos, S. M. A. (2012). Influência dos determinantes do envelhecimento ativo entre idosos mais idosos. Texto Contexto Enfermagem, 21(1), 167-176.

- Fernandes, M. G. M., Barbosa, K. T. F., Oliveira, F. M. L. R., Rodrigues, M. M. D. \& Santos, K. F. O. (2014). Risco de quedas evidenciado por idosos atendidos num ambulatório de geriatria. Revista Eletrônica de Enfermagem, 16(2), 297-303.

- Fernandes, M. G. M. \& Garcia, L. G. O. (2010). O sentido da velhice para homens e mulheres idosos. Saúde Sociedade, 19(4), 771-783.

- Ferreira, O. G. L., Maciel, S. C., Silva, A. O., Santos, W. S., \& Moreira, M. A. S. P. (2010). O envelhecimento ativo sob o olhar de idosos funcionalmente independentes. Revista da Escola de Enfermagem da USP, 44(4), 1065-1069.

- Ferreira, O. G. L., Maciel, S. C., Costa, S. M. G., Silva, A. O. \& Moreira, M. A. S. P. (2012). Envelhecimento ativo e sua relação com a independência funcional. Texto Contexto Enfermagem, 21(3), 513-518.

- Galicioli, T. G. P., Lopes, E. S. de L. \& Rabelo, D. F. (2012). Superando a viuvez na velhice: o uso de estratégias de enfrentamento. Revista Temática Kairós Gerontologia, 15(4), 225-237.

- Geib, L. T. C. Determinantes sociais da saúde do idoso. (2012). Ciência e Saúde Coletiva, 17 (1), 123-133.

- Gonçalves, L. H. T., Nassar, S. M., Daussy, M. F. S., Santos, S. M. A. \& Alvarez, A. M. (2011). O convívio familiar do idoso na quarta idade e seu cuidador. Ciência Cuidado e Saúde, 10(4), 746-754.

- Instituto Brasileiro de Geografia e Estatística (2011). Estatísticas do registro civil 2011. Brasília, BR.

- Instituto Brasileiro de Geografia e Estatística (2012). Síntese de indicadores sociais: Uma análise das condições de vida da população brasileira 2012. Brasília, BR.

- Instituto Brasileiro de Geografia e Estatística (2013). Estatísticas da população 2013. Brasília, BR.

- Nogueira, A., Alberto, M., Cardoso, G. A. \& Barreto, M. A. M. (2012). Risco de queda nos idosos: educação em saúde para melhoria da qualidade de vida. Revista prá$x i s, 4(8), 77-82$.

- Oliveira, L. P. B. A. \& Menezes, M. P. (2011). Representações de fragilidade para idosos no contexto da estraté- 
gia saúde da família. Texto Contexto Enfermagem, 20(2), 301-309.

- Oliveira, M. B., Silva, N. M., Teixeira, K. M. D. (2013). Transferências financeiras: os idosos como suporte econômico familiar. VI Workshop de Analise Ergonômica do trabalho.

- Organização Mundial da Saúde (2005). Envelhecimento ativo: uma política de saúde. Brasília, Brasil.

- Reis, R. H., Schwartz, E., Lima, L. M., Lange, C., Feijó, A. M. \& Duarte, G. C. (2014). Autopercepção de saúde de idosos usuários de um serviço de oncologia. Revista Eletrônica de Enfermagem, 16(3), 612-618.

- Ribeiro, O. \& Paúl, C. (2011). Envelhecimento Ativo: Manual de Envelhecimento Ativo. Lisboa: Lidel.

- Sant'anna, C. F., Cezar-Vaz, M. R., Cardoso, L. S., Erdmann, A. L. \& Soares, J. F. S. (2010). Determinantes sociais de saúde: características da comunidade e trabalho das enfermeiras na saúde da família. Revista Gaúcha de Enfermagem. 31(1), 92-99.

- Santos, J. P. \& Paes, N. A. (2014). Associação entre condições de vida e vulnerabilidade com a mortalidade por doenças cardiovasculares de homens idosos do nordeste. Revista. Brasileira de. Epidemiologia, 17(2), 407-420.

- Sebastião, C. \& Albuquerque, C. Envelhecimento e de-

Tabela 1 - Distribuição das variáveis de identificação dos idosos atendidos em um Centro de Atenção Integral à Saúde dos Idosos, João Pessoa - PB, Brasil, 2015. (n = 224). pendência. Estudo sobre os impactes da dependência de um membro idoso na família e no cuidador principal. Revista Kairós Gerontologia, 14(4), 25-49.

- Secretaria de Direitos Humanos (2012). Dados sobre o envelhecimento no Brasil. Brasília, BR.

- Silva, S. P. C. \& Menandro, M. C. S. (2013). Representações de idosos sobre a vacina da gripe. Ciência e Saúde Coletiva, 18(8), 2179-2188.

- Sthal, H. C., Berti, H. W. \& Palhares, V.C. (2010). Caracterização de idosos internados em enfermaria de pronto-socorro quanto à vulnerabilidade social e programática. Revista Escola Anna Nery, 14(4), 697-704.

- Traldi, L. P. Z (2013). Perfil e atitudes de idosos e cuidadores atendidos na estratégia saúde da família. (Dissertação não publicada). FMRP - USP, São Paulo.

- Vicente, F. R (2012). Validação de conteúdo de um instrumento para avaliação multidimensional do envelhecimento ativo. (Dissertação não publicada). Universidade Federal de Santa Catarina, Florianópolis.

- Vicente, F. R. \& Santos, S. M. A. (2013). Avaliação multidimensional dos determinantes do envelhecimento ativo em idosos de um município de Santa Catarina. Texto Contexto Enfermagem, 22(2), 370-378.

\begin{tabular}{|c|c|c|c|}
\hline Vari $\square$ vel & Categorias & $\mathbf{n}$ & $\%$ \\
\hline \multirow[t]{3}{*}{ Faixa et $\llbracket$ ria } & 60 a 69 & 135 & 60,3 \\
\hline & 70 a 79 anos & 72 & 32,1 \\
\hline & 80 ou mais & 17 & 7,6 \\
\hline \multirow[t]{2}{*}{ Sexo } & Feminino & 153 & 68,3 \\
\hline & Masculino & 71 & 31,7 \\
\hline \multirow[t]{4}{*}{ Religi ${ }^{\circ}$} & Cat $\square$ ica & 148 & 66,1 \\
\hline & Evang lica & 69 & 30,8 \\
\hline & Esp rita & 04 & 1,8 \\
\hline & Nenhuma & 03 & 1,3 \\
\hline \multirow[t]{4}{*}{ Estado Civil } & Casado & 89 & 39,7 \\
\hline & Vi־vo & 68 & 30,4 \\
\hline & Solteiro & 37 & 16,5 \\
\hline & Divorciado & 30 & 13,4 \\
\hline
\end{tabular}


Tabela 2 - Distribuição das variáveis econômicas das idosas atendidas em um Centro de Atenção Integral à Saúde dos Idosos, João Pessoa - PB, Brasil, 2015. (n

\begin{tabular}{lrrr}
\hline \multicolumn{1}{c}{ Vari $\square$ vel } & Categorias & n & \% \\
\hline \multirow{3}{*}{ Renda } & Menos que 1 sal rio m nimo* & 21 & 9,4 \\
& Entre 1 e 3 sal rios m nimos & 192 & 85,7 \\
& Entre 3 e 5 sal rios m nimos & 11 & 4,9
\end{tabular}

Benef cios sociais

$\begin{array}{rrr}\text { Aposentadoria } & 145 & 64,7 \\ \mathrm{~N} \_ \text {recebe } & 32 & 14,3 \\ \text { Fundo de pens } \square & 20 & 8,9 \\ \text { Aux lios sociais } & 12 & 5,4 \\ \text { Recebe dois ou mais benef cios } & 12 & 5,4 \\ \text { Previd } \square \text { ncia privada } & 3 & 1,3\end{array}$

Trabalho n

remunerado

$\begin{array}{rrr}\text { Nenhum } & 142 & 63,4 \\ & & \\ \text { Em casa } & 113 & 50,4 \\ \text { Jardim e/ou horta } & 78 & 34,8 \\ \text { Cuidador } & 54 & 24,1 \\ \text { Volunt rio em igreja e/ou escolas } & 39 & 17,4\end{array}$

*Sal $\sqcap$ rio m nimo no per odo de coleta de dados $=\mathrm{R} \$ 788,00$.

\begin{tabular}{|c|c|c|c|}
\hline Vari vel & Categorias & $\mathbf{n}$ & $\%$ \\
\hline \multirow[t]{4}{*}{ Escolaridade } & 1 a 3 anos & 48 & 21,4 \\
\hline & 4 a 8 anos & 77 & 34,4 \\
\hline & 9 ou mais & 76 & 33,9 \\
\hline & Nenhum & 23 & 10,3 \\
\hline \multirow[t]{7}{*}{ Viol $\square$ ncia e maus tratos } & Nenhum & 107 & 47,8 \\
\hline & Roubado & 120 & 53,6 \\
\hline & Abandono familiar & 17 & 7,6 \\
\hline & Abandono da Sociedade & 09 & 4,0 \\
\hline & For $\sqsubset$ ado a abrir $\mathrm{m} \sqsubset \mathrm{o}$ dos rendimentos & 03 & 1,3 \\
\hline & For ado a realizar atividades & 02 & 0,9 \\
\hline & Abuso Sexual & 01 & 0,4 \\
\hline \multirow[t]{2}{*}{ Arranjo familiar } & Com familiares & 179 & 79,9 \\
\hline & Sozinho & 45 & 20,1 \\
\hline
\end{tabular}

Redes de apoio

$\begin{array}{rrr}\text { Igreja } & 149 & 66,5 \\ \text { Fora de casa } & 84 & 37,5 \\ \text { Dentro de casa } & 83 & 37,1 \\ \text { Grupo de conviv } \llbracket \text { ncia } & 56 & 25,0 \\ \text { Associa } \square \text { es } & 11 & 4,9\end{array}$

Tabela 3 - Distribuição dos determinantes sociais das idosas atendidas em um Centro de Atenção Integral à Saúde dos Idosos, João Pessoa - PB, Brasil, 2015. $(\mathrm{n}=224)$ 
Tabela 4 - Distribuição das variáveis relacionadas aos determinantes de ambiente físico das idosas atendidas em um Centro de Atenção Integral à Saúde dos Idosos, João Pessoa - PB, Brasil, 2015. $(n=224)$

\begin{tabular}{|c|c|c|c|}
\hline Vari $\square$ vel & Categorias & $\mathbf{n}$ & $\%$ \\
\hline \multirow[t]{5}{*}{ Saneamento b $b$ sico } & ç gua e rede pluvial & 123 & 54,9 \\
\hline & ç gua e fossa & 86 & 38,4 \\
\hline & ç gua e rede ao c $\sqsubset \mathrm{u}$ aberto & 11 & 4,9 \\
\hline & $\mathrm{N} \curvearrowleft$ sabe & 03 & 1,3 \\
\hline & Po $\sqsubset$ e fossa & 01 & 0,4 \\
\hline \multirow[t]{4}{*}{ Coleta lixo } & Coletado & 207 & 92,4 \\
\hline & $\mathrm{C} \square \mathrm{u}$ aberto & 14 & 6,3 \\
\hline & Parte coletada e parte queimada & 02 & 0,9 \\
\hline & Queimado ou enterrado & 01 & 0,4 \\
\hline \multirow[t]{6}{*}{ Propriedade da resid $\square$ cia } & $\operatorname{Pr} \sqcap$ pria e quarto privativo & 181 & 80,8 \\
\hline & Casa alugada em qual mora o idoso & 17 & 7,6 \\
\hline & $\operatorname{Pr} \sqsubset$ pria e quarto dividido & 09 & 4,0 \\
\hline & Casa do filho e quarto privativo & 08 & 3,6 \\
\hline & Casa alugada com outros familiares & 07 & 3,1 \\
\hline & Casa do filho e quarto dividido & 02 & 0,9 \\
\hline \multirow[t]{9}{*}{ Condi $\square$ es das casas* } & Bem iluminada & 181 & 80,8 \\
\hline & Degraus ou escadas & 63 & 28,1 \\
\hline & Tapetes & 53 & 23,7 \\
\hline & Animais dentro de casa & 47 & 21,0 \\
\hline & Animais fora de casa & 39 & 17,4 \\
\hline & Pisos irregulares ou escorregadios & 26 & 11,6 \\
\hline & Moveis para trope ar & 21 & 9,4 \\
\hline & Nenhum & 13 & 5,8 \\
\hline & Banheiro fora de casa & 01 & 0,4 \\
\hline
\end{tabular}

*Possibilidade de mais de uma op $\square$ o de resposta 